\title{
Trastornos eosinofílicos del tubo digestivo
}

\author{
Claudia Martínez-Camacho* \\ Departamento de Endoscopia, Hospital de Especialidades CMN Siglo XXI, IMSS, Ciudad de México, México
}

Los trastornos eosinofílicos gastrointestinales se consideran trastornos inflamatorios crónicos inmunomediados y desencadenados por alérgenos, caracterizados por la infiltración patológica de eosinófilos en esófago, estómago, intestino delgado o colon que conduce a la disfunción del órgano afectado con presencia de síntomas clínicos, los cuales dependen del sitio afectado y de la profundidad de la invasión de la pared intestinal (mucosa, muscular, subserosa). Los síntomas son muy diversos y pueden incluir: disfagia, impactación de alimentos, dolor abdominal, cólico, náuseas, vómitos, saciedad temprana, diarrea y pérdida de peso ${ }^{1}$.

Se consideran poco comunes, a excepción de la esofagitis eosinofílica (EoE), ya que se ha incrementado el reporte mundial de casos. Un metaanálisis calculó una incidencia global de 3.7/100,000/año (IC 95\%: 1.7-6.5), la incidencia en adultos fue alta, 7.0/100,000/ año y en niños 5.1/100,000/año. La prevalencia global combinada de 22.7/100,000 (IC 95\%: 12.4-36.0), en adultos $43.4 / 100,000$ (IC 95\%: 22.5-71.2) y niños 29.5 (IC 95\%: 17.5-44.7) aunque hubo heterogenicidad sustancial en estas estimaciones ${ }^{2,3}$.

En la DDW 2021 se presentaron 37 trabajos principalmente en ciencias básicas y respuesta a nuevos tratamientos propuestos. Analizaremos 3 trabajos enfocados al área de la endoscopia gastrointestinal. DeIlon ES, et al., con Optimization of eosinophilic gastritis/ duodenitis detection requires evaluation of multiple high-powered fields in each of 8 gastric and 4 duodenal biopsies: Analiysis from a randomized trial. La gastritis eosinofílica (EG) y la duodenitis eosinofílica (EoD) pueden estar infradiagnosticadas debido a la falta de criterios de diagnóstico estandarizados, incluido el número óptimo de biopsias. Evaluaron las tasas de diagnóstico y definieron el número de biopsias necesarias para la detección de EG/EoD, utilizando datos de cribado de un ensayo controlado aleatorizado de lirentelimab (AK002) en pacientes con sintomatología gastrointestinal de moderada a severa (malestar y doIor abdominal, distensión, saciedad temprana, perdida del apetito, náuseas, vómito y diarrea), a quienes se realizó endoscopia digestiva alta con un toma de biopsias gástricas y duodenales, seguidas de evaluación histopatológica que incluía el recuento de eosinófilos (eos); los criterios histopatológicos para el diagnóstico fueron $\geq 30$ eos por campo de alta potencia (hpf) en $\geq$ 5 hpf gástricos para EG y $\geq 3$ hpf duodenales para EoD. De un total de 88 pacientes que se les realizo endoscopia, 72 cumplieron criterios para EG/EoD, de los cuales fueron 10 (14\%) pacientes con EG, 35 (49\%) con $E G+E o D$ y 27 (38\%) con EoD, hubo una alta variación en la concentración de eosinófilos entre las biopsias, con una media $( \pm \mathrm{DE})$ de $2.6( \pm 2.3)$ de 8 biopsias gástricas y $2.2( \pm 1.2)$ de 4 biopsias duodenales recolectados por sujeto. Cada biopsia gástrica y duodenal adicional recolectada durante la endoscopia resultó en un aumento incremental en el porcentaje de casos de EG/EoD diagnosticados. Concluyendo que la alta tasa de detección en pacientes no diagnosticados previamente y la irregularidad de la eosinofilia gástrica y duodenal sugiere que un protocolo de biopsias de un mínimo de 8 gástricas y 4 duodenales aumentará el rendimiento diagnóstico en $\mathrm{EG}$ y EoD ${ }^{4}$.

\section{Correspondencia:}


Hirano, et al. presentaron Endoscopic appearance does not predict the presence of histologic eosinophilic gastritis among symptomatic adults, indicating that collection and evaluation of biopsies should occur regardless of endoscopic appearance: Analysis from a randomized trial. El objetivo fue caracterizar los hallazgos endoscópicos en sujetos con EG y compararlos con los de individuos sintomáticos sin EG utilizando datos de cribado de un ensayo controlado aleatorizado de lirentelimab (AK002). Los sujetos que cumplieron con los criterios de síntomas se sometieron a una endoscopia superior, durante la cual los investigadores puntuaron prospectivamente las características gástricas de acuerdo con el sistema de referencia endoscópica de gastritis eosinofílica (EG-REFS), esta puntúa 7 parámetros (granularidad, erosión, nodularidad, eritema, friabilidad, pliegues, estenosis pilórica) en 3 localizaciones gástricas (fondo, cuerpo, antro). Los criterios histológicos de EG ( $\geq 30 \mathrm{eos} / \mathrm{hpf}$ en $\geq 5 \mathrm{hpf}$ gástricos). En resultados de 72 pacientes que cumplieron criterios de síntomas se encontró que 45 (63\%) tenían EG (con o sin EoD), las alteraciones endoscópicas estaban ausentes o eran leves en la mayoría de los sitios, pero se identificaron con mayor frecuencia en el antro, las más comunes fueron eritema (86\%), erosiones $(64 \%)$ y granularidad $(57 \%)$, en pacientes $\sin$ EG pero con EoD eritema (70\%), erosiones (37\%) y granularidad (44\%), en aquellos sintomáticos sin EG ni EoD fue eritema (75\%), erosiones (25\%) y granularidad (44\%), por lo que la puntuación total de EG-REFS no se correlacionó con EG o EoD histológica. Concluyendo que los hallazgos endoscópicos no son específicos de EG y es importante la toma de biopsias sistematizada y contar los eosinófilos tisulares en pacientes sintomáticos, independientemente de los hallazgos endoscópicos ${ }^{5}$.

Beveridge, et al. presentaron Pilot feasibility study of encapsulated balloon cell sampling device compared to endoscopy with biopsies for the evaluation of eosinophiliec esophagitis. La endoscopia superior (EGD) con biopsias es el método para evaluar la actividad de la enfermedad de EoE, el balón encapsulado (EB) es un dispositivo mínimamente invasivo, el cual se traga, se infla el balón, se jala y colecta las células, se desinfla el balón y retira. El objetivo fue evaluar la viabilidad y seguridad de la EB en comparación con las biopsias endoscópicas en EoE. Se incluyeron 6 pacientes con EoE y se utilizó el EB antes de la endoscopia. Resultados: 5 (83\%) pacientes completaron el $\mathrm{EB}$, en 3 pacientes de endoscopia se reportaron eosinófilos (5, 50 y 56 eos/hpf) y en ellos se detectaron eosinófilos con el EB (40, 230 y 27 eos/hpf) respectivamente, 3 pacientes no tenían eosinófilos en las biopsias y 2 de ellos tampoco tuvieron con el EB y 1 paciente no completo el EB, 2 (33\%) pacientes refirieron dolor de garganta. En este estudio piloto el EB es una alternativa mínimamente invasiva factible, segura y bien tolerada a las biopsias endoscópicas para evaluar la actividad de la enfermedad, se necesita determinar la precisión y características del rendimiento en una población mayor ${ }^{6}$.

En la UEG Week 2020 se presentaron 21 trabajos de EoE que evaluaron evolución clínica y respuesta a los diversos tratamientos. Navarro, et al. presentaron Fibrotic features in the eosinophilic esophagitis Endoscopic Reference Score in the diagnosis and response to proton-pump inhibitor therapy. El sistema de clasificación Endoscopic Reference Score de la EoE (EREFS) está validado y puede ayudar a identificar características inflamatorias (edema, exudados y surcos) y fibróticas (anillos y estrías) en el esófago de pacientes con EoE. Investigaciones recientes han sugerido que los inhibidores de bomba de protones (IBP) tienen un impacto limitado en los procesos de EoE subepiteliales como la fibrosis, el objetivo fue investigar el efecto potencial de la terapia con IBP en la reducción de las características endoscópicas de la EoE y la reversión de las fibróticas después de un curso a corto plazo. Se incluyeron pacientes que recibieron terapia con IBP para inducir remisión de la EoE y se excluyeron pacientes sometidos a dilatación esofágica. Los cambios en la puntuación general de la EREFS y la subpuntuación fibrótica se compararon entre los pacientes que respondieron y los que no respondieron al tratamiento con IBP. Resultados de 169 pacientes con EoE, 96 pacientes en la endoscopia basal presentaban anillos y 26 estenosis esofágica. La puntuación de EREFS se redujo significativamente solo en los que respondieron a los IBP $2.59( \pm 1.73)$ vs. $1.06( \pm 1.01)(p<0.001)$, sin cambios encontrados entre los que no respondieron $2.85( \pm 1.68)$ vs. 2.12 $( \pm 1.86)$, la remisión histológica profunda no proporcionó reducciones adicionales en la subpuntuaciones de anillos y estenosis, pero la puntuación fibrótica combinada mostro una disminución casi significativa 1.43 \pm 1.25 vs. $0.93 \pm 0.89(p=0.05)$, concluyendo que la terapia con IBP eficaz para la EoE se asocia a una mejora endoscópica significativa y reduce significativamente las características endoscópicas de la fibrosis esofágica a corto plazo?. 


\section{Financiamiento}

La presente investigación no ha recibido ayudas específicas provenientes de agencias del sector público, sector comercial o entidades sin ánimo de lucro.

\section{Conflicto de intereses}

Los autores declaran no tener conflicto de intereses.

\section{Bibliografía}

1. Gonsalves N. Eosinophilic gastrointestinal disorders. Clinic Rev Allerg Immmunol. 2019;57(2):272-85.

2. Godwin B, Wilkins B, Muir A. EoE disease monitoring: Where we are and wherw we are going. Ann Allergy Asthma Immunol. 2020;124:240-7.
3. Arias A, Perez-Martinez I, Tenias JM. Systematic review with meta-analysis: the incidence and prevalence of eosinophilic eosophagitis in children and adults in population-based studies. Aliment Pharmacol Ther. 2016;43:3-15.

4. Dellon ES, Gonsalves N, Rothenberg M, Hirano I, Chehade M, Peterson KA, et al. Optimization of eosinophilic gastritis/duodenitis detectión requires evaluation of multiple high-powered fields in each of 8 gastric and 4 duodenal biopsies: Analysis from a randomized trial [Internet]. Allakos, Scientific Presentations; mayo de 2021. Disponible en: https:// www.allakos.com/file.cfm/59/docs/Dellon_et_al_DDW_May_2021.pdf

5. Hirano I, Chehade M, Gonsalves N, Rothenberg M, Peterson KA, Falk GW, et al. Endoscopic appearance does not predict the presence of histologic eosinophilic gastritis among symptomatic adults, indicating that collection and evaluation of biopsies should occur regardless of endoscopic appearance: Analysis from a randomized trial [Internet]. Allakos, Scientific Presentations; mayo de 2021. Disponible en: https:/l www.allakos.com/file.cfm/59/docs/Hirano_et_al_DDW_May_2021.pdf

6. Beveridge $C$, Thanawala S, Hunt $D$, et al. Pilot feasibility study of encapsulated ballon cell sampling device compared to endoscopy with biopsies for the evaluation of eosinophilic esophagitis. DDw 2021 \# 180.

7. Navarro P, Laserna Mendieta EJ, Guagnozzi D, Casabona S, Perelló A Savarino $E$, et al. Fibrotic features in the eosinophilic esophagitis Endoscopic Reference Score in the diagnosis and response to proton-pump inhibitor therapy. Digestive and Liver Disease. Preimpresión disponible online 10/06/2021: https://doi.org/10.1016/.dld.2021.05.025 